Review

\title{
Metal Oxide Nanomaterial QNAR Models: Available Structural Descriptors and Understanding of Toxicity Mechanisms
}

\author{
Jiali Ying ${ }^{1,2, \dagger}$, Ting Zhang $1,2, \dagger, *$ and Meng Tang $1,2, *$
}

1 Key Laboratory of Environmental Medicine Engineering, Ministry of Education, School of Public Health, Southeast University, Nanjing 210009, China; E-Mail: yingjiali1020@163.com Jiangsu key Laboratory for Biomaterials and Devices, Southeast University, Nanjing 210009, China

$\dagger$ These authors contributed equally to this work.

* Authors to whom correspondence should be addressed;

E-Mails: zhangting@seu.edu.cn (T.Z.); tm@seu.edu.cn (M.T.);

Tel./Fax: +86-025-83272564 (T.Z. \& M.T.).

Academic Editor: Jiye Fang

Received: 11 September 2015 / Accepted: 3 October 2015 / Published: 12 October 2015

\begin{abstract}
Metal oxide nanomaterials are widely used in various areas; however, the divergent published toxicology data makes it difficult to determine whether there is a risk associated with exposure to metal oxide nanomaterials. The application of quantitative structure activity relationship (QSAR) modeling in metal oxide nanomaterials toxicity studies can reduce the need for time-consuming and resource-intensive nanotoxicity tests. The nanostructure and inorganic composition of metal oxide nanomaterials makes this approach different from classical QSAR study; this review lists and classifies some structural descriptors, such as size, cation charge, and band gap energy, in recent metal oxide nanomaterials quantitative nanostructure activity relationship (QNAR) studies and discusses the mechanism of metal oxide nanomaterials toxicity based on these descriptors and traditional nanotoxicity tests.
\end{abstract}

Keywords: nanotoxicology; metal oxide; quantitative structure activity relationship (QSAR); descriptor; toxicity mechanisms 


\section{Introduction}

Nanotechnology involves the study of the synthesis, characterization, and properties of nanomaterials [1]. The emergence of nanotechnology has led to innovations in many areas of electronics, energy management, structural materials, functional surfaces, construction, information technology, pharmaceuticals, and medical devices. The largest share of the manufacturing and application market among the different nanomaterials belongs to metal oxide nanomaterials, they are already used in some consumer products, such as $\mathrm{TiO}_{2}$ in paints and $\mathrm{ZnO}$ in sunscreen products [2]. Moreover, since the $1950 \mathrm{~s}$, millions of tons of materials such as carbon black, $\mathrm{SiO}_{2}, \mathrm{TiO}_{2}, \mathrm{and}_{\mathrm{Al}_{2} \mathrm{O}_{3}}$ have been produced per year for use in paints, catalysts, and surfactants [3]. Nanomaterials can release into the environment during production, transport, and use [4], and metal oxide nanomaterials are no exception [5]. The divergent published toxicology data on metal oxide nanomaterials makes it difficult to determine whether there is a risk associated with metal oxide nanomaterial exposure [2]. The small size and large specific surface area of metal oxide nanomaterials endows them with high chemical reactivity and intrinsic toxicity [6]. Therefore, most nanotoxicity studies have concentrated on metal oxide nanomaterials, and different metal oxide nanomaterials have different toxic effects according to previous studies [7-17].

However, performing toxicology tests for each metal oxide nanomaterial is time consuming and resource intensive; therefore, researchers are developing computational nanotoxicology methods, such as quantitative structure activity relationship (QSAR) modeling, to predict the toxicity of metal oxide nanomaterials. Such predictions would allow researchers to prioritize toxicology tests on real metal oxide nanomaterials [18]. QSAR modeling was originally a computational chemistry method; it is known as nano quantitative structure activity relationship (nano-QSAR) [19] or quantitative nanostructure activity relationship (QNAR) [20] modeling in nanotoxicology. In this review, we use the term QNAR modeling. QSAR modeling attempts to correlate a set of structural parameters (which are called molecular descriptors) that describe a chemical compound with its biological activity. The QSAR modeling procedure includes the selection of molecular descriptors and toxicology endpoint descriptors [21,22], as well as construction, validation [23-25], and evaluation [19] of the model. It is not our purpose to discuss every procedure in QNAR modeling here, which have been reviewed by many other researchers [3,19,20,22,26-30]. Current reviews about QNAR research in the past five years have been listed in Table 1. Instead, we focus on the molecular descriptors in this review because, as we know, we use metal oxide nanomaterial QNAR method to high-throughput screening of the toxicity of metal oxide nanomaterials and to guide the production of metal oxide nanomaterials, the most important steps to improve QNAR models are the selection of structural descriptors, to help readers moving forward in the implementation of QNAR, we listed the available structural descriptors and further analyzed the key issues in metal oxide QNAR study, and to the best of our knowledge, they have not been reviewed before. 
Table 1. Quantitative nanostructure activity relationship (QNAR) reviews in the past five years.

\begin{tabular}{|c|c|c|}
\hline Title & Summary & Ref \\
\hline $\begin{array}{l}\text { No time to lose-high throughput } \\
\text { screening to assess nanomaterial safety }\end{array}$ & $\begin{array}{l}\text { This review aims to provide a comprehensive } \\
\text { introduction to the high throughput/content } \\
\text { screening methodology employed for safety } \\
\text { assessment of engineered nanomaterials, } \\
\text { including data analysis and prediction of } \\
\text { potentially hazardous material properties. }\end{array}$ & {$[3]$} \\
\hline $\begin{array}{l}\text { Exploring QNAR modeling as a tool for } \\
\text { predicting biological effects of } \\
\text { manufactured nanoparticles }\end{array}$ & $\begin{array}{l}\text { The review discusses major approaches for model } \\
\text { building and validation using both experimental } \\
\text { and computed properties of nanomaterials by } \\
\text { considering two different categories of } \\
\text { nanomaterials datasets:(i) those comprising } \\
\text { nanomaterials with diverse metal cores and } \\
\text { organic decorations;(ii) those involving } \\
\text { nanomaterials possessing the same core. }\end{array}$ & [20] \\
\hline $\begin{array}{l}\text { Predictive models for nanotoxicology: } \\
\text { Current challenges and future opportunities }\end{array}$ & $\begin{array}{l}\text { The review aims to provide researchers strategies } \\
\text { for directing research towards predictive models } \\
\text { and the ancillary benefits of such research. } \\
\text { The purpose of this review is to provide a } \\
\text { summary of recent key advances in the field of }\end{array}$ & [19] \\
\hline $\begin{array}{l}\text { Applying quantitative structure-activity } \\
\text { relationship approaches to nanotoxicology: } \\
\text { Current status and future potential }\end{array}$ & $\begin{array}{l}\text { QNAR modelling, to identify the major gaps in } \\
\text { research required to accelerate the use of QSAR } \\
\text { methods, and to provide a road map for future } \\
\text { research needed to achieve QSAR models useful } \\
\text { for regulatory purposes. }\end{array}$ & {$[26]$} \\
\hline $\begin{array}{l}\text { Advancing risk assessment of engineered } \\
\text { nanomaterials: Application of } \\
\text { computational approaches }\end{array}$ & $\begin{array}{l}\text { The purpose of this review is to present the } \\
\text { current state of knowledge related to the risks of } \\
\text { the engineered nanoparticles and to assess the } \\
\text { potential of efficient expansion and development } \\
\text { of new approaches, which are offered by } \\
\text { application of theoretical and computational } \\
\text { methods, applicable for evaluation of } \\
\text { nanomaterials. }\end{array}$ & {$[27]$} \\
\hline $\begin{array}{l}\text { Nano(Q)SAR: Challenges, pitfalls and } \\
\text { perspectives }\end{array}$ & $\begin{array}{l}\text { This article aims to identify some of the pitfalls } \\
\text { and challenges associated with (Q)NARs. Three } \\
\text { major barriers were identified: the need to } \\
\text { improve quality of experimental data in which the } \\
\text { models are developed from, the need to have } \\
\text { practical guidelines for the development of the } \\
\text { (Q)NAR models and the need to standardise and } \\
\text { harmonise activities for the purpose of regulation. }\end{array}$ & [28] \\
\hline
\end{tabular}

In classical QSAR modeling, these molecular descriptors typically relate to the steric and electronic properties of the chemical compound and can be measured experimentally or computationally. Classical QSAR studies usually begin from the stage of molecular modeling and descriptor 
calculation $[31,32]$. There are many ways to classify classical molecular descriptors, such as theoretical molecular descriptors include 0D-descriptors, 1D-descriptors, 2D-descriptors, 3D-descriptors, and 4D-descriptors [33]. However, the molecular descriptor of a nanomaterial is different from classical QSAR molecular descriptors, and we should give it a proper name "structural descriptor" in QNAR modeling because nanomaterials are no longer simple chemical compounds. The metal oxide nanomaterials QSAR study should be classified by two categories, (i) coated metal oxide nanomaterials QSAR study: the selection of coated metal oxide nanomaterials structural descriptors is always the structural descriptor of its organic surface modification; and (ii) bare metal oxide nanomaterials QSAR study: the selection of bare metal oxide nanomaterials structural descriptors is a challenge in metal oxide QNAR model-building because of its inorganic composition and nanostructure. For the first type of study, the surface modification of coated metal nanomaterials was considered to be the key factor to influence its toxicity [34,35]; therefore, we can refer to it as organic chemicals QSAR study. However, the relationship between the surface modification group of metal oxide nanomaterials and nanotoxicity is not the focus of this article. In this review, we emphasize on bare metal oxide nanomaterials QSAR study; all we discuss below is about bare metal oxide nanomaterials. Based on previous studies [36-41], we have classified the metal oxide nanomaterial structural descriptors as experimental descriptors (describing the morphological structural properties and physicochemical properties of metal oxide nanomaterials) and theoretical descriptors (describing the constitutional properties, electronic or thermodynamic properties); in addition, we summarize how to obtain these structural descriptors and introduce some novel structural descriptors used in existing metal oxide QNAR models. Finally, and also drawing on recent nanotoxicity studies, we propose mechanisms for metal oxide nanomaterial toxicity from the existing QNAR models based on these descriptors. We hope that this article will be useful for researchers, especially for toxicologists, in performing metal oxide nanomaterial QNAR research.

\section{Experimental Descriptors}

Metal oxide nanomaterial experimental descriptors are usually experimental measurements, such as size distribution, agglomeration state, shape, porosity, surface area, and chemical composition. Metal oxide nanomaterials exhibit different properties than bulk materials or isolated molecules. Knowing the morphology and physicochemical properties of metal oxide nanomaterials can open new exciting opportunities for modeling metal oxide nanomaterial characteristics through the use of the QSAR approach, so it is meaningful to classify these experimental measurements based on morphological structural properties and physicochemical properties which provide researches different views to select structural descriptors. Here we use size, shape, porosity, and surface area as morphological structural properties in addition to zeta potential and surface modification $[6,7,16,42,43]$.

\subsection{Morphological Structural Properties}

Metal oxide nanomaterials can be characterized by different sizes, shapes, porosities, surface areas, crystals, and aggregation; these characteristics are usually classified as physico-chemical properties, and we classify them as morphological structural properties in this review. Many techniques are complementary and used together to support the data. Various techniques such as scanning electron 
microscopy (SEM), transmission electron microscopy (TEM), dynamic light scattering (DLS), static light scattering (SLS), and atomic force microscopy (AFM) are available for primary size, size distribution, agglomeration state, porosity, and shape characterization [42-45]. Brunauer-Emmett-Teller (BET) surface area measurements can be used to measure a given amount of metal oxide nanomaterial based on the gas adsorption surface area, as well as the porosity of the samples [44,46-49]. X-ray diffraction (XRD) analysis is a classical crystallographic structure measurement technique that can be used for metal oxide nanomaterials with respect to the arrangements of atoms in the bulk as well as on the surface. This technique uses the patterns, positions, intensities, and shapes of the peaks to elicit the atomic structure of the samples. Moreover, small angle X-ray scattering (SAXS) can also be used to reveal the crystallographic properties of metal oxide nanoparticles [50-52]. Gajewicz [40] calculated 11 image descriptors (area $(A)$, volume $(V)$, surface diameter $(D s)$, volume/mass diameter $(\mathrm{d} V / m)$, volume/surface diameter (dSauter), aspect ratio $x$ (AR_x), aspect ratio $y\left(\mathrm{AR} \_y\right)$, porosity $X(\mathrm{P} X)$, porosity $Y(\mathrm{PY})$, sphericity $(\Psi)$, and circularity (fcirc)) based on TEM microscopic images, which can reflect the size, size distribution, shape, porosity, and surface area for all 18 nanometer-sized metal oxides. These descriptors were not included in the final model; however, they provided us with a method to quantify the image characterization. Liu et al. [53] used nanoparticle primary size and volume fraction (in solution) as two of the structural descriptors to build a classification nano-SAR model, and the classification accuracy for both internal validation and external validation was $100 \%$. Size has also been used in metal oxide nanomaterials QNAR models [54-58].

\subsection{Physicochemical Properties}

Physicochemical properties include zeta potential, acidity coefficient $(\mathrm{pK}$ ), relaxivities, isoelectric point, and surface charge. The zeta potential of nanomaterials is measured by light-scattering electrophoresis or electro-acoustophoresis, and the isoelectric point (IEP) can be determined using electrophoretic mobility measurements $(\mathrm{EPM})$. $\mathrm{pK}_{\mathrm{a}}$ can be measured by titration in various suspending media. Liquid contact angle assessment reveals additional information about surface chemistry, charge, and energy. Elemental speciation and redox state can be measured by Raman, X-ray absorption fine structure (XAFS), X-ray absorption near edge structure (XANES), and nuclear magnetic resonance (NMR), and electronic, magnetic, and photonic properties can be analyzed by small- and wide-angle X-ray scattering (SAXS), Mossbauer, electron spin resonance (ESR), Raman, and ultraviolet-visible spectroscopy (UV-Vis). "Dustiness" or tendency to aerosolize, can be measured by a scanning mobility particle sizer (SMPS). Charge density, $\mathrm{pK}_{\mathrm{a}}$, point of zero charge (PZC), and ionization fraction can be tested by direct titration in various suspending media [3]. The total concentration of the metal oxide can be measured by X-ray fluorescence (XRF), and the concentration of soluble metal can be measured by inductively coupled plasma mass spectrometry (ICP-MS) conjugation with ultrafiltration [59]. Band gap energy (Eg) can be measured by UV-Vis spectroscopy [60]. UV-Vis spectroscopy can also be used as an alternative method to roughly evaluate nanoparticle sedimentation. Redox potential can be measured using an oxidation reduction potential (ORP) electrode probe [61]. Duffin et al. [62] proposed approaches that presented the possibility of modeling the potential toxicity of metal nanoparticles and nuisance dusts based on the inflammatory response under an instilled surface area dose. The zeta potential and IEP have been included in a metal oxide nanomaterial 
nanoSAR model [53]. Singh et al. [56] used relativities $\left(R_{1}, R_{2}\right)$ and zeta potential as four of the model's parameters to predict the toxicity of 51 different NMs (with diverse metal cores) in four cell lines. Fourches used the zeta potential and relativities to build a nanoQSAR model [58]. Cho et al. [63] correlated the percentage of granulocytes of rats exposed to metal oxide nanomaterials with the zeta potential in physiological saline $(\mathrm{pH}$ 5.6) of these metal oxide nanomaterials. Main experimental structural descriptors used in some metal oxide QNAR models was reviewed in Table 2.

Table 2. Main experimental structural descriptors used in some metal oxide (Q)NAR models.

\begin{tabular}{|c|c|c|c|c|c|c|}
\hline \multicolumn{6}{|c|}{ Experimental structural descriptors } & \multirow[b]{2}{*}{ Ref } \\
\hline Size & $\begin{array}{l}\text { Volume } \\
\text { fraction }\end{array}$ & $\begin{array}{c}\text { Zeta } \\
\text { potential }\end{array}$ & $\begin{array}{c}\text { Relativities } \\
R_{1}\end{array}$ & $\begin{array}{c}\text { Relativities } \\
\boldsymbol{R}_{\mathbf{2}}\end{array}$ & IEP & \\
\hline \multirow[t]{5}{*}{$\sqrt{ }$} & $\sqrt{ }$ & $\sqrt{ }$ & & & $\sqrt{ }$ & Liu et al. [53] \\
\hline & & $\sqrt{ }$ & $\sqrt{ }$ & $\sqrt{ }$ & & Epa et al. [54] \\
\hline & & $\sqrt{ }$ & $\sqrt{ }$ & $\sqrt{ }$ & & Singh et al. [56] \\
\hline & & $\sqrt{ }$ & $\sqrt{ }$ & $\sqrt{ }$ & & Fourches et al. [58] \\
\hline & & $\sqrt{ }$ & & & & Cho et al. [63] \\
\hline
\end{tabular}

\section{Theoretical Descriptors}

Theoretical descriptors usually involve applications of quantum chemical or molecular simulation methods that have been proven to be reliable and efficient means to predict molecular properties. Classical theoretical molecular descriptors are derived from a symbolic representation of the molecule and can be further classified according to the different types of molecular representation. Similar to classical molecular descriptors, theoretical descriptors are an important part of metal oxide nanomaterials QSAR study, but with their own special properties; metal oxide nanomaterials consist of metal oxide crystal structures, and we should, thus, first determine their crystal structure.

\subsection{Constitutional Properties}

Constitutional properties are simple molecular descriptors that can be obtained from the periodic table of elements, chemical handbooks, and molecular formulae, such as the molecular weight of the molecule, weight of the atom, number of molecular descriptors, and cation charge. This is a cost-effective approach to obtain molecular descriptors without depending on quantum software. $\mathrm{Hu}$ et al. [6] reported that the cytotoxicity of seven metal oxide nanoparticles was observed to be correlated with their cation charges. Kar et al. [39] used metal electronegativity ( $\chi$ ), the charge of the metal cation corresponding to a given oxide $(\chi \mathrm{Ox})$, atomic number and valence electron number of the metal as simple molecular descriptors to develop QSAR models for predicting the cytotoxicity of 17 metal oxide nanomaterials to Escherichia coli. Moreover, the period of the nanoparticle metal has also been used in a metal oxide nanomaterials cytotoxicity classification model [53].

\subsection{Electronic Properties}

Electronic properties are properties relate to energy and are the most widely used properties in metal oxide QNAR model building. Some electronic properties of metal oxides can be used as descriptors, 
such as band gap and valence gap energy. However, most metal oxide nanomaterials are difficult to dissolve in solution, they are nano-size in water; so the unusual properties of nanoparticles, such as their hardness, high yield strength, flexibility, rigidity, and ductility, are related to their high surface-to-volume ratio, and the quantum size effect and macro-quantum tunneling effect are attributable to their nano-size [64]. Therefore, it is necessary to determine the metal oxide properties on the nanoscale. Fortunately, some contributions have highlighted that the most significant size-dependent changes of some properties of nanoparticles are observed below approximately $5 \mathrm{~nm}$; thus, changes in nanomaterials properties between 15 and $90 \mathrm{~nm}$ can be ignored [65,66]. Burello et al. [67] proposed a theoretical framework of a descriptor calculation that is related to the oxidative stress potential of oxide nanoparticles based on the assumption that a particle has a diameter larger than 20-30 $\mathrm{nm}$ and no surface states in the band gap. Since some quantum-chemical properties change with the diameter of the nanoparticle [68], another approach involves software-based calculations (e.g., CODESSA [69], DRAGON [70], CAChe [71], and MOE [72]). However, from a quantum chemistry viewpoint, nanoparticles (10-1000 $\AA$ ) are very large systems [68]; therefore, it is necessary to maximally simplify the molecular models used for geometry optimization and/or calculation of the molecular properties to reflect the variability of the nanoparticles' structures [19]. Gajewicz et al. [68] observed that molecular properties change with size in two ways based on MOPAC software; one way is that the parameters vary non-linearly with the considered crystal size (e.g., $\mathrm{ZnO}, 11 \AA$ A) until they reach a saturation point (type A), and the other way is that the parameters vary linearly with the crystal size. Therefore, we can use the saturation point to determine the type A properties and use the linear equation to determine the type B properties with calculations based on many atoms.

Puzyn et al. [41] used the $\Delta \mathrm{H}_{\mathrm{Me}}$ descriptor that represents the enthalpy of formation of a gaseous cation with the same oxidation state as that in the metal oxide structure as a parameter to construct the toxicity prediction model for Escherichia coli. Gajewicz et al. [40] combined experimental-theoretical studies to develop an interpretative QNAR model to predict the toxicity of 18 nano-metal oxides to the HaCaT cell line. A simple and statistically significant QSAR model was successfully developed for the dark group based on two descriptors, the absolute electronegativity of the metal and of the metal oxide. Similarly, the best correlation was obtained to predict the photo-induced toxicity of metal oxide nanomaterials using two descriptors, the molar heat capacity and average of the alpha and beta lowest unoccupied molecular orbital (LUMO) energies of the metal oxide [37]. Liu et al. [73] used the atomization energy of the metal oxide, atomic mass of metal nanoparticles, conduction band energy of the nanoparticles, metal oxide ionization energy, and metal oxide electronegativity as descriptors to construct several metal oxide nanomaterial toxicity classification models. Main theoretical structural descriptors used in some metal oxide QNAR models are reviewed in Table 3. 
Table 3. Main theoretical structural descriptors used in some metal oxide QNAR models.

\begin{tabular}{ll}
\hline Structural descriptors & Ref \\
\hline $\begin{array}{l}\text { Cation charges } \\
\begin{array}{l}\text { The absolute electronegativity of the metal and of the metal } \\
\text { oxide, the molar heat capacity and average of the alpha and } \\
\text { beta LUMO }\end{array}\end{array}$ & Hu Pathakoti et al. [37] \\
$\begin{array}{l}\text { Metal electronegativity, the charge of the metal cation, atomic } \\
\text { number, valence electron number of the metal }\end{array}$ & Kar et al. [39] \\
$\begin{array}{l}\text { Standard enthalpy of formation of metal oxide nanocluster, } \\
\begin{array}{l}\text { Mulliken's electronegativity } \\
\text { The enthalpy of formation of a gaseous cation with the same } \\
\text { oxidation state as that in the metal-oxide structure }\end{array}\end{array}$ & Gajewicz et al. [40] \\
\hline
\end{tabular}

\section{Other Novel Descriptors}

Actually, except these structural descriptors listed above, some new methods appeared to select more comprehensive structural descriptors for researchers.

\subsection{Liquid Drop Model}

The other novel descriptor is the "liquid drop" model (LDM). The LDM was applied to describe the supramolecular structure of nanoparticles [36]. It is beneficial to use a combination of descriptors that reflect the structure of the nanoparticles for the different levels of organization: from a single molecule to a supramolecular ensemble of molecules, to the interactions of nanomaterials with biological systems. Sizochenko et al. [36] established an LDM for 17 metal oxide nanomaterials to predict the toxicity toward Escherichia coli and HaCaT cells.

\subsection{QSAR-Perturbation Approach Based Descriptors}

Luan et al. [38] used the descriptor DDV (ca.) to characterize the physicochemical properties, as well as the types of cells against which the metal oxide nanomaterials were tested. The descriptors represent their relative degrees of importance in the QSAR-perturbation model. This model can simultaneously predict the cytotoxicity of different nanoparticles against several mammalian cell lines while considering different times of exposure of the cell lines, as well as the chemical composition of the nanoparticles, their size, the conditions under which the size was measured, and their shape.

\subsection{Optimal SMILE-Based Descriptor}

SMILES is a sequence of symbols which represent the molecular architecture. It has been applied in QSAR study of carbon nanotubes [74] and some organic chemicals [75]. It has been proven it can be applied to metal oxide nanomaterials QNAR model building. Toropova et al. [76] constructed a model to predict the membrane damage caused by a group of zinc oxide and titanium oxide nanomaterials; Toropova et al. [77] built up a model for prediction of dark cytotoxicity and photo-induced cytotoxicity of metal oxide nanoparticles to the bacteria Escherichia coli. The advantages and disadvantages of different structural descriptors in metal oxide QNAR studies can be seen in Table 4. 
Table 4. Advantages and disadvantages of different structural descriptor types in metal oxide QNAR studies.

\begin{tabular}{|c|c|c|}
\hline Descriptors type & Advantages & Disadvantages \\
\hline $\begin{array}{l}\text { morphological structural } \\
\text { properties }\end{array}$ & $\begin{array}{l}\text { directly relate to the characteristics of } \\
\text { metal oxide nanomaterials, easy to } \\
\text { explain the toxicity mechanism }\end{array}$ & $\begin{array}{l}\text { measuring error, some of the } \\
\text { properties are difficult to } \\
\text { quantitate }\end{array}$ \\
\hline physicochemical properties & $\begin{array}{l}\text { directly relate to characteristics of } \\
\text { metal oxide nanomaterials, easy to } \\
\text { explain the toxicity mechanism }\end{array}$ & measuring error \\
\hline constitutional properties & easy to obtain & $\begin{array}{l}\text { characteristics of metal } \\
\text { oxide nanomaterial are not } \\
\text { included }\end{array}$ \\
\hline $\begin{array}{l}\text { electronic properties or } \\
\text { thermodynamic properties }\end{array}$ & $\begin{array}{l}\text { easy to obtain, easy to explain the } \\
\text { toxicity mechanism }\end{array}$ & $\begin{array}{l}\text { the calculation system is } \\
\text { relatively small }\end{array}$ \\
\hline novel descriptors & $\begin{array}{l}\text { directly relate to the characteristics of } \\
\text { metal oxide nanomaterials, easy to } \\
\text { explain the toxicity mechanism }\end{array}$ & $\begin{array}{l}\text { the calculation method is } \\
\text { complex }\end{array}$ \\
\hline
\end{tabular}

\section{Understanding Toxicity Mechanism(s) from Existing QNAR Models}

In traditional nanotoxicity studies, the toxicity of metal oxide nanomaterials is related to their size, size distribution, surface area, shape, zeta potential, surface charge, aggregation state, and the extent of ion detachment from the surface [2]. Karlsson et al. [10] discovered that nano-sized copper oxide was much more toxic than micro-sized copper oxide toward A549 cells. Hamilton et al. [78] observed that the alteration of anatase titanium dioxide nanomaterials into a fiber structure of greater than $15 \mu \mathrm{m}$ was highly toxic and initiated an inflammatory response by alveolar macrophages. Fröhlich et al. [79] demonstrated that charge density and hydrophobicity are equally important in non-phagocytic cell ingestion, but that the cellular uptake of cationic NPs is higher than that of anionic NPs in these cells; phagocytic cells, however, preferentially take up anionic nanomaterials. Rogers [80] observed that body length and progeny count decreased and organismal stress increased in Caenorhabditis elegans when exposed to aggregated cerium oxide $(0-17.21 \mathrm{ug} / \mathrm{mL})$. Heinlaan et al. [5] noticed that the metal ions detached from the surface were responsible for the toxicity of nano-sized zinc oxide and nano-sized copper oxide in Vibrio fisheri bacteria. However, in recent metal nano-QSAR studies, size, size distribution, shape, surface area, zeta potential, surface charge, and aggregation state were not the key factors that affected the toxicity of the metal oxide nanomaterials, and some descriptors related to the release of ions detached from the metal oxide were often included in the models. Puzyn et al. [41] observed that the particle size of the metal oxide nanomaterials did not affect the antibacterial activity in their model; however, the descriptor that represented the enthalpy of formation of the gaseous cation having the same oxidation state as that in the metal oxide structure was used to build a satisfactory model that can be applied to predict the EC50 of E. coli. Kar et al. [39] used some simple molecular descriptors, such as metal electronegativity, charge of the metal cation corresponding to a given oxide, atomic number and valence electron number of the metal, to develop QSAR models for the EC50 of metal oxide nanoparticles toward E. coli. Pathakoti et al. [37] used the absolute electronegativity of the 
metal atom and the absolute electronegativity of the metal oxide to predict EC50 of metal oxide nanomaterials to $E$. coli in dark conditions, and used the literature molar heat capacity of the metal oxide at $298.15 \mathrm{~K}$ and the average of the alpha and beta lowest unoccupied molecular orbital energy of the metal oxide to predict the photo-induced toxicity of the metal oxide nanomaterials. Gajewicz et al. [40] used two descriptors (the enthalpy of formation of the metal oxide nanocluster representing a fragment of the surface and the Mulliken's electronegativity of the cluster) to predict the median lethal concentration (LC50) of metal oxide nanomaterials toward HaCaT cells. The primary size was only included in a few metal oxide toxicity classification nanoSAR models [53,73].

We proposed a possible toxicity mechanism of metal oxide nanomaterials to explain these conflicts. Size, size distribution, shape, surface area, zeta potential, surface charge, and aggregation state are not direct factors that cause cell inhibition, cell death, or cell apoptosis. They can affect the cellular uptake of metal oxide nanomaterials by van der Waals forces, steric interactions, and electrostatic charges [3]. It can be performed by directly affecting the function of the cell membrane [81,82], or indirectly performed by some chemical activities, such as influencing the formation of protein corona [83], than finally influence their ability to enter cell. After entering the cell, metal oxide stability is an important factor that causes toxic effects, such as ions detaching from the surface of metal oxide nanomaterials, inducing ROS generation and then causing a series of oxidative stress reactions related to cell viability, cell apoptosis and cell death. In addition, we cannot ignore extracellular ROS and ions release induced toxicity $[83,84]$. From the characterization aspect, surface charge is usually reported as the zeta potential, which takes into account the electric potential in the interfacial double layer as well as the $\mathrm{pK}_{\mathrm{a}}$ of the particle [85]. The surface charge of nanomaterials is particularly interesting as it is one of the factors that controls the dispersion and aggregation of engineered nanomaterials as well as affecting cellular uptake [86]. Surface charge may determine binding sites for receptors, affect the dispersion and aggregation of the particles, and affect the capacity to produce reactive oxygen species (ROS) [79]. Stable metal oxides do not exhibit any toxic effects, whereas metallic nanomaterials that have a redox potential may be cytotoxic and genotoxic [87]. The stability is found to be of prime importance to dictate $\mathrm{ZnO}$ QDs toxicity either towards the ROS generation or towards the liberation of metal [88]. Heavy metal ions induce oxidative stress and inflammatory responses and electron-hole pair generation during photoactivation, leading to free-radical generation [3], which is consistent with the results from some metal oxide nanomaterials QNAR studies.

In a 2008 NATO workshop, experts proposed that toxicity may occur via one or a combination of four possible mechanisms [89]. The first mechanism suggested that the release of the chemical constituents from the nanomaterials leads to toxicity, such as the release of toxic anions. The second mechanism suggested that the size and shape of the particle produces steric hindrances or interferes with some important binding sites. The third mechanism of toxicity is related to the surface properties of the materials, such as local electric fields, electronic semi-conductance and charge densities. Finally, the fourth mechanism of toxicity involves the capacity of nanomaterials to act as vectors for transporting other toxic chemicals to sensitive tissues. The results of the QNAR study and nanotoxicity test study are consistent with these mechanisms. We combined metal oxide nanoparticle toxicity mechanisms with a recent metal oxide nanomaterials QNAR study that included a toxicity test, as shown in Figure 1. 


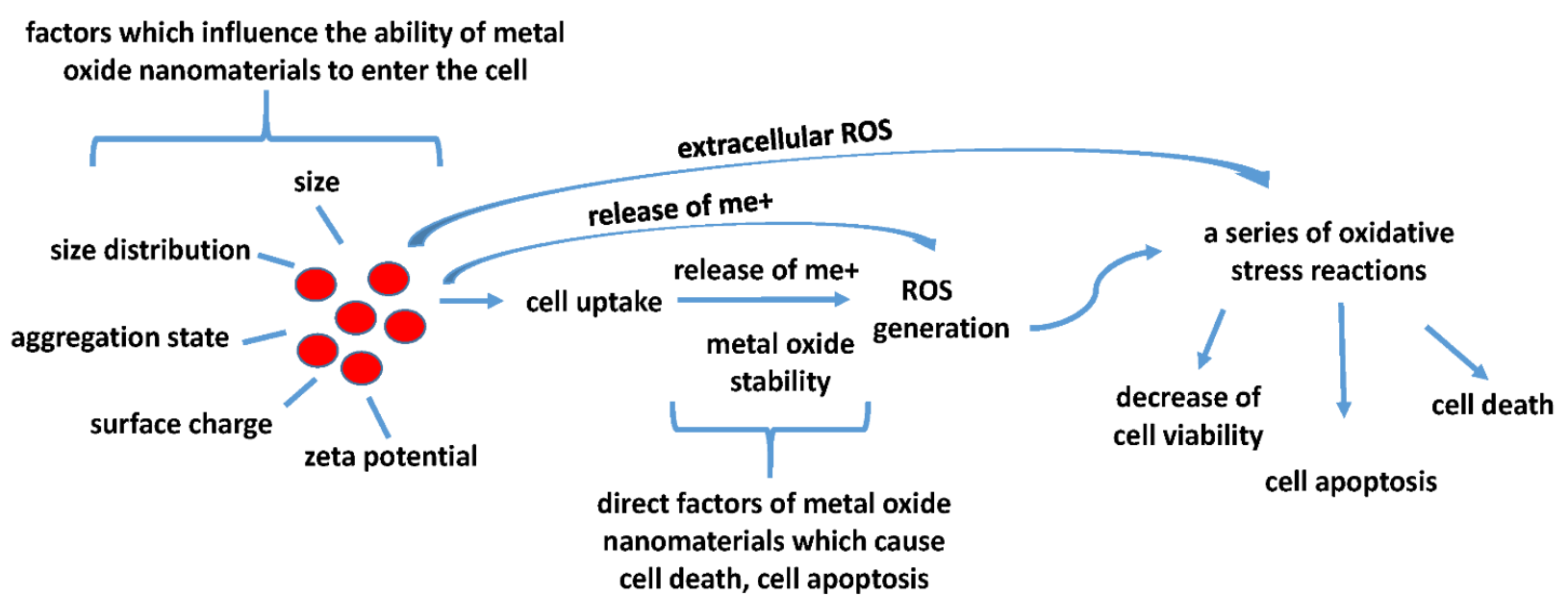

: metal oxide nanomaterials

Figure 1. Characteristics such as size, size distribution, aggregation state, surface charge, and zeta potential can affect the cellular uptake of metal oxide nanomaterials. After entering the cell, metal oxide stability is an important factor that causes toxic effects, such as ions detaching from the surface of metal oxide nanomaterials, inducing ROS generation and then causing a series of oxidative stress reactions related to cell viability, cell apoptosis and cell death. In addition, extracellular ROS and released ions can also induce a series of oxidative stress reactions.

\section{Conclusions and Outlook}

Metal oxide nanomaterials QNAR modeling is a comprehensive task that requires the combined efforts of toxicologists, inorganic chemists, materials scientists, and statisticians. We aim to provide readers available structural descriptors and the method to obtain these structural descriptors after reading this review. Recent studies have demonstrated that QNAR modeling is an effective and accurate tool to assess the biological activity and toxic potential of metal oxide nanomaterials in a short period with reduced cost. On one side, we use QNAR model to predict the toxicity of metal oxide nanomaterials; however, there were unavoidable problems in recent QNAR models, which are discussed here. First, most of the recent models were based on in vitro toxicity tests; there are few metal oxide nanomaterials QNAR models based on in vivo toxicity tests or model organisms, even though the toxicity data should involve multiple species in different environmental systems. Unavoidably, it was difficult to obtain some quantitative toxicity data for these organisms, such as median lethal dose (LD50) and lethal concentration 50 (LC50), because the toxicity of some metal oxide nanomaterials was very low. Second, the elucidation of the mechanism of toxicity appears to be a complex problem, with many studies reporting conflicting results. The toxicity tests should be cross-validated in multiple laboratories; reliable experimental data are necessary for the successful development of new QNAR, which requires researchers to work together with the QSAR community to provide reliable and useful data to better understand the toxicity mechanism of metal oxide nanomaterials. Third, most of the existing metal oxide QNAR models are based on limited data, which may cause some statistical errors; we should extend the types of metal oxide nanomaterials studied to reduce these errors. Meanwhile validation is vital to ensure that the predictive ability of the model is 
not caused by chance factors, but there are no standardized validation metrics [28]. On the other side, we use QNAR models to design safer metal oxide nanomaterials based on their structural descriptors, but some of the structural descriptors, such as constitutional structural descriptors, are not associated with characteristics of metal oxide nanomaterials, so it is necessary to select structural descriptors which are directly relate to the characteristics of metal oxide nanomaterials. Additionally, the issue of chemical doping which is reported as a potential factor causing toxicity, should also be considered in future QNAR studies. Only reliable and meaningful QNAR models can be applied to the high-throughput screening of the toxicity of metal oxide nanomaterials and to guide the production of metal oxide nanomaterials.

\section{Acknowledgments}

This work was financially supported by the National Natural Science Foundation of China (No. 81172697, 81302461 and 81473003); National Important Project on Scientific Research of China (No. 2011CB933404); and Specialised Research Fund for the Doctoral Program of Higher Education (No. 20130092120062).

\section{Author Contributions}

All authors discussed the contents of the review manuscript. Y.J.L. wrote the text of the manuscript and made the Figure and Tables for paper. Z.T contributed to revise the manuscript. Modifications and the proof reading of the manuscript has also been done by Z.T. The manuscript is prepared under the supervision of T.M.

\section{Conflicts of Interest}

The authors declare that there are no conflicts of interest.

\section{References}

1. Fernández-Garcia, M.; Rodriguez, J.A. Encyclopedia of Inorganic and Bioinorganic Chemistry: Metal Oxide Nanoparticles; John Wiley \& Sons, Ltd: New Yor, NY, USA, 2011; pp.1-3.

2. Teow, Y.; Asharani, P.V.; Hande, M.P.; Valiyaveettil, S. Health impact and safety of engineered nanomaterials. Chem. Commun. 2011, 47, 7025-7038.

3. Damoiseaux, R.; George, S.; Li, M.; Pokhrel, S.; Ji, Z.; France, B.; Xia, T.; Suarez, E.; Rallo, R.; Madler, L.; et al. No time to lose-High throughput screening to assess nanomaterial safety. Nanoscale 2011, 3, 1345-1360.

4. Gottschalk, F.; Nowack, B. The release of engineered nanomaterials to the environment. J. Exp. Med. 2011, 13, 1145-1155.

5. Heinlaan, M.; Ivask, A.; Blinova, I.; Dubourguier, H.C.; Kahru, A. Toxicity of nanosized and bulk $\mathrm{ZnO}, \mathrm{CuO}$ and $\mathrm{TiO}_{2}$ to bacteria Vibrio fischeri and crustaceans Daphnia magna and Thamnocephalus platyurus. Chemosphere 2008, 71, 1308-1316.

6. Hu, X.; Cook, S.; Wang, P.; Hwang, H.M. In vitro evaluation of cytotoxicity of engineered metal oxide nanoparticles. The Sci. Total Environ. 2009, 407, 3070-3072. 
7. Mahmoudi, M.; Hofmann, H.; Rothen-Rutishauser, B.; Petri-Fink, A. Assessing the in vitro and in vivo toxicity of superparamagnetic iron oxide nanoparticles. Chem. Rev. 2012, 112, 2323-2338.

8. Zuzana, M.; Alessandra, R.; Lise, F.; Maria, D. Safety assessment of nanoparticles cytotoxicity and genotoxicity of metal nanoparticles in vitro. J. Biomed. Nanotechnol. 2011, 7, 20-21.

9. Mahmoudi, M.; Simchi, A.; Milani, A.S.; Stroeve, P. Cell toxicity of superparamagnetic iron oxide nanoparticles. J. Colloid. Interf. Sci. 2009, 336, 510-518.

10. Karlsson, H.L.; Gustafsson, J.; Cronholm, P.; Moller, L. Size-dependent toxicity of metal oxide particles-A comparison between nano- and micrometer size. Toxicol. Lett. 2009, 188, 112-118.

11. Park, E.J.; Choi, J.; Park, Y.K.; Park, K. Oxidative stress induced by cerium oxide nanoparticles in cultured BEAS-2B cells. Toxicology 2008, 245, 90-100.

12. Lundqvist, M.; Stigler, J.; Elia, G.; Lynch, I.; Cedervall, T.; Dawson, K.A. Nanoparticle size and surface properties determine the protein corona with possible implications for biological impacts. Proc. Natl. Acad. Sci. USA 2008, 105, 14265-14270.

13. Hanna, L.; Karlsson, P.C.; Johanna, G.; Lennart, M. Copper oxide nanoparticles are highly toxic: A comparison between metal oxide nanoparticles and carbon nanotubes. Chem. Res. Toxicol. 2008, 21, 1726-1732.

14. Wang, J.; Zhou, G.; Chen, C.; Yu, H.; Wang, T.; Ma, Y.; Jia, G.; Gao, Y.; Li, B.; Sun, J.; et al. Acute toxicity and biodistribution of different sized titanium dioxide particles in mice after oral administration. Toxicol. Lett. 2007, 168, 176-185.

15. Warheit, D.B.; Webb, T.R.; Sayes, C.M.; Colvin, V.L.; Reed, K.L. Pulmonary instillation studies with nanoscale $\mathrm{TiO}_{2}$ rods and dots in rats: Toxicity is not dependent upon particle size and surface area. Toxicol. Sci. 2006, 91, 227-236.

16. Jeng, H.A.; Swanson, J. Toxicity of metal oxide nanoparticles in mammalian cells. J. Environ. Sci. Health A 2006, 41, 2699-2711.

17. Hussain, S.M.; Hess, K.L.; Gearhart, J.M.; Geiss, K.T.; Schlager, J.J. In vitro toxicity of nanoparticles in BRL 3A rat liver cells. Toxicol. In Vitro 2005, 19, 975-983.

18. Burello, E.; Worth, A. Computational nanotoxicology: Predicting toxicity of nanoparticles. Nat. Nanotechnol. 2011, 6, 138-139.

19. Clark, K.A.; White, R.H.; Silbergeld, E.K. Predictive models for nanotoxicology: Current challenges and future opportunities. Regul. Toxicol. Pharm. 2011, 59, 361-363.

20. Fourches, D.; Pu, D.; Tropsha, A. Exploring quantitative nanostructure-activity relationships (QNAR) modeling as a tool for predicting biological effects of manufactured nanoparticles. Comb. Chem. High Throughput Screen. 2011, 14, 217-225.

21. Djurisic, A.B.; Leung, Y.H.; Ng, A.M.; Xu, X.Y.; Lee, P.K.; Degger, N.; Wu, R.S. Toxicity of metal oxide nanoparticles: Mechanisms, characterization, and avoiding experimental artefacts. Small 2015, 11, 26-44.

22. Landsiedel, R.; Ma-Hock, L.; Kroll, A.; Hahn, D.; Schnekenburger, J.; Wiench, K.; Wohlleben, W. Testing metal-oxide nanomaterials for human safety. Adv. Mater. 2010, 22, 2601-2627.

23. Ma, W.J.; Ruys, A.J.; Mason, R.S.; Martin, P.J.; Bendavid, A.; Liu, Z.; Ionescu, M.; Zreiqat, H. DLC coatings: Effects of physical and chemical properties on biological response. Biomaterials 2007, 28, 1620-1628. 
24. Kroll, A.; Dierker, C.; Rommel, C.; Hahn, D.; Wohlleben, W.; Schulze-Isfort, C.; Gobbert, C.; Voetz, M.; Hardinghaus, F.; Schnekenburger, J. Cytotoxicity screening of 23 engineered nanomaterials using a test matrix of ten cell lines and three different assays. Part. Fibre Toxicol. 2011, 8, 1-19.

25. Lauerent, S.; Saei A.A.; Behzdi S.; Panahifar A. ; Mahmoudi M. Superpa ramagnetic iron oxide nanoparticles for delivery of therapeutic agents: Opportunities and challenges. Expert Opin. Drug Deliv. 2014, 11, 1449-1470.

26. Winkler, D.A.; Mombelli, E.; Pietroiusti, A.; Tran, L.; Worth, A.; Fadeel, B.; McCall, M.J. Applying quantitative structure-activity relationship approaches to nanotoxicology: Current status and future potential. Toxicology 2013, 313, 15-23.

27. Gajewicz, A.; Rasulev, B.; Dinadayalane, T.C.; Urbaszek, P.; Puzyn, T.; Leszczynska, D.; Leszczynski, J. Advancing risk assessment of engineered nanomaterials: Application of computational approaches. Adv. Drug Deliv. Rev. 2012, 64, 1663-1693.

28. Tantra, R.; Oksel, C.; Puzyn, T.; Wang, J.; Robinson, K.N.; Wang, X.Z.; Ma, C.Y.; Wilkins, T. Nano(Q)SAR: Challenges, pitfalls and perspectives. Nanotoxicology 2015, 9, 636-642.

29. Karelson, M. The QSPR/QSAR Approach for the Prediction of Properties of Fullerence Derivatives. Ph.D. Thesis, University of Tartu, Estonia, 2011.

30. Ronald, H.; White, M.S.T. Assessing Nanomaterial Risks: Current Challenges and Future Opportunities for Risk Assessment. In Proceedings of the American Chemistry Council-Long Range Research Initiative Workshop: Navigating Obstacles towards Modernizing Risk Assessment, Washington, DC, USA, 24 February 2010.

31. Yuan, J.; Pu, Y.; Yin, L. Prediction of binding affinities of PCDDs, PCDFs and PCBs using docking-based comparative molecular similarity indices analysis. Environ. Toxicol. Pharmacol. 2014, 38, 1-7.

32. Yuan, J.; Pu, Y.; Yin, L. Docking-based three-dimensional quantitative structure-activity relationship (3D-QSAR) predicts binding affinities to aryl hydrocarbon receptor for polychlorinated dibenzodioxins, dibenzofurans, and biphenyls. Environ. Toxicol. Pharmacol. 2013, 32, 1453-1458.

33. Todeschini, R.; Conso, V. Handbook of Molecular Descriptors; Wiley-VCH: Weinheim, Germany, 2000; pp. 927-933.

34. Toropova, A.P.; Toropov, A.A.; Benfenati, E.; Korenstein, R.; Leszczynska, D.; Leszczynski, J. Optimal nano-descriptors as translators of eclectic data into prediction of the cell membrane damage by means of nano metal-oxides. Environ Sci. Pollut. Res. 2015, 22, 745-757.

35. Liu, R.; Rallo, R.; Bilal, M.; Cohen, Y. Quantitative structure-activity relationships for cellular uptake of surface-modified nanoparticles. Comb. Chem. High Throughput Screen. 2015, 18, 365-375.

36. Sizochenko, N.; Rasulev, B.; Gajewicz, A.; Kuz'min, V.; Puzyn, T.; Leszczynski, J. From basic physics to mechanisms of toxicity: The "liquid drop" approach applied to develop predictive classification models for toxicity of metal oxide nanoparticles. Nanoscale 2014, 6, 13986-13993.

37. Pathakoti, K.; Huang, M.J.; Watts, J.D.; He, X.; Hwang, H.M. Using experimental data of Escherichia coli to develop a QSAR model for predicting the photo-induced cytotoxicity of metal oxide nanoparticles. J. Photochem. Photobiol. B 2014, 130, 234-240. 
38. Luan, F.; Kleandrova, V.V.; Gonzalez-Diaz, H.; Ruso, J.M.; Melo, A.; Speck-Planche, A.; Cordeiro, M.N. Computer-aided nanotoxicology: Assessing cytotoxicity of nanoparticles under diverse experimental conditions by using a novel QSTR-perturbation approach. Nanoscale 2014, 6, 10623-10630.

39. Kar, S.; Gajewicz, A.; Puzyn, T.; Roy, K.; Leszczynski, J. Periodic table-based descriptors to encode cytotoxicity profile of metal oxide nanoparticles: A mechanistic QSTR approach. Ecotoxicol. Environ. Saf. 2014, 107, 162-169.

40. Gajewicz, A.; Schaeublin, N.; Rasulev, B.; Hussain, S.; Leszczynska, D.; Puzyn, T.; Leszczynski, J. Towards understanding mechanisms governing cytotoxicity of metal oxides nanoparticles: Hints from nano-QSAR studies. Nanotoxicology 2015, 9, 313-325.

41. Puzyn, T.; Rasulev, B.; Gajewicz, A.; Hu, X.; Dasari, T.P.; Michalkova, A.; Hwang, H.M.; Toropov, A.; Leszczynska, D.; Leszczynski, J. Using nano-QSAR to predict the cytotoxicity of metal oxide nanoparticles. Nat. Nanotechnol. 2011, 6, 175-178.

42. Shaw, B.J.; Handy, R.D. Physiological effects of nanoparticles on fish: A comparison of nanometals versus metal ions. Environ. Int. 2011, 37, 1083-1097.

43. Han, D.W.; Hong, S.C.; Lee, J.H.; Lee, J.; Kim, H.Y.; Park, J.Y.; Cho, J.; Lee, J. Subtle cytotoxicity and genotoxicity differences in superparamagnetic iron oxide nanoparticles coated with various functional groups. Int. J. Nanomed. 2011, 6, 3219-3231.

44. Jensen, H.; Perdersen, H.; Jørgensen, J.E.; Pedersen, J.S.; Joensen, K.D.; Iversen, S.B.; Søgaard, E.G. Determination of size distributions in nanosized powders by TEM, XRD, and SAXS. J. Exp. Nanosci. 2006, 1, 355-373.

45. Bootz, A.; Vogel, V.; Schubert, D.; Kreuter, J. Comparison of scanning electron microscopy, dynamic light scattering and analytical ultracentrifugation for the sizing of poly (butyl cyanoacrylate) nanoparticles. Eur. J. Pharm. Biopharm. 2004, 57, 369-375.

46. Jillavenkatesa, A.; Kelly, J.F. Nanopowder characterization: Challenges and future directions. J. Nanopart. Res. 2002, 4, 463-468.

47. Warheit, D.B.; Frame, S.R. Characterization and reclassification of titanium dioxide-related pulmonary lesions. J. Occup. Environ. Med. 2006, 48, 1308-1313.

48. Seto, T.; Kawakami, Y.; Suzuki, N.; Hirasawa, M.; Kano, S.; Aya, N.; Sasaki, S.; Shimura, H. Evaluation of morphology and size distribution of silicon and titanium oxide nanoparticles generated by laser ablation. J. Nanopart. Res. 2001, 3, 185-191.

49. Fisker, R.; Carstensen, J.M.; Hansen, M.F.; Bødker, F.; Mørup, S. Estimation of nanoparticle size distributions by image analysis. J. Nanopart. Res. 2000, 2, 267-277.

50. Chu, B.; Liu, T. Characterization of nanoparticles by scattering techniques. J. Nanopart. Res. 2000, 2, 29-41.

51. Itoh, Y.; Lenggoro, W.; Okuyama, K.; Mädler, L.; Pratsinis, S.E. Size tunable synthesis of highly crystalline $\mathrm{BaTiO}_{3}$ nanoparticles using salt-assisted spray pyrolysis. J. Nanopart. Res. 2003, 5, 191-198.

52. Harano, A.; Shimada, K.; Okubo, T.; Sadakata, M. Crystal phases of $\mathrm{TiO}_{2}$ ultrafine particles prepared by laser ablation of solid rods. J. Nanopart. Res. 2002, 4, 215-219.

53. Liu, R.; Rallo, R.; George, S.; Ji, Z.; Nair, S.; Nel, A.E.; Cohen, Y. Classification nanoSAR development for cytotoxicity of metal oxide nanoparticles. Small 2011, 7, 1118-1126. 
54. Epa, V.C.; Burden, F.R.; Tassa, C.; Weissleder, R.; Shaw, S.; Winkler, D.A. Modeling biological activities of nanoparticles. Nano Lett. 2012, 12, 5808-5812.

55. Burello, E.; Worth, A.P. QSAR modeling of nanomaterials. WIREs Nanomed. Nanobiotechnol. 2011, 3, 298-306.

56. Singh, K.P.; Gupta, S. Nano-QSAR modeling for predicting biological activity of diverse nanomaterials. RSC Adv. 2014, 4, 13215-13230.

57. Wang, X.Z.; Yang, Y.; Li, R.; McGuinnes, C.; Adamson, J.; Megson, I.L.; Donaldson, K. Principal component and causal analysis of structural and acute in vitro toxicity data for nanoparticles. Nanotoxicology 2014, 8, 465-476.

58. Fourches, D.; Pu, D.; Tassa, C.; Weissleder, R.; Shaw, S.Y.; Mumper, R.J.; Tropsha, A. Quantitative nanostructure-activity relationship modeling. ACS Nano 2010, 4, 5703-5712.

59. Horie, M.; Fujita, K.; Kato, H.; Endoh, S.; Nishio, K.; Komaba, L.K.; Nakamura, A.; Miyauchi, A.; Kinugasa, S.; Hagihara, Y.; et al. Association of the physical and chemical properties and the cytotoxicity of metal oxide nanoparticles: Metal ion release, adsorption ability and specific surface area. Metallomics 2012, 4, 350-360.

60. Zhang, H.; Ji, Z.X.; Xia, T.; Meng, H.; Low-Kam, C.; Liu, R.; Pokhrel, S.; Lin, S.; Wang, X.; Liao, Y.P.; et al. Use of metal oxide nanoparticle band gap to develop a predictive paradigm for oxidation stress and acute pulmonary inflammation. ACS Nano 2012, 6, 4349-4368.

61. Tantra, R.; Cackett, A.; Peck, R.; Gohil, D.; Snowden, J. Measurement of redox potential in nanoecotoxicological investigations. J. Toxicol. 2012, 2012, 270651.

62. Duffin, R.; Tran, L.; Brown, D.; Stone, V.; Donaldson, K. Proinflammogenic effects of low-toxicity and metal nanoparticles in vivo and in vitro: Highlighting the role of particle surface area and surface reactivity. Inhal. Toxicol. 2007, 19, 849-856.

63. Cho, W.S.; Duffin, R.; Thielbeer, F.; Bradley, M.; Megson, I.L.; Macnee, W.; Poland, C.A.; Tran, C.L.; Donaldson, K. Zeta potential and solubility to toxic ions as mechanisms of lung inflammation caused by metal/metal oxide nanoparticles. Toxicol. Sci. 2012, 126, 469-477.

64. Puzyn, T.; Cronin, M.T.D.; Leszczynski, J. Recent advances in QSAR studies: Methods and applications. Cronin. Eds. 2010, 8, 383-410.

65. Kukreja, L.; Barik, S.; Misra, P. Variable band gap ZnO nanostructures grown by pulsed laser deposition. J. Cryst. Growth 2004, 105, 531-535.

66. Qu, Z.W.; Kroe, G.J. Theoretical study of the electronic structure and stability of titanium dioxide clusters $\left(\mathrm{TiO}_{2}\right)_{n}$ with $n=1-9$. J. Phys. Chem. B 2006, 110, 8998-9007.

67. Burello, E.; Worth, A.P. A theoretical framework for predicting the oxidative stress potential of oxide nanoparticles. Nanotoxicology 2011, 5, 228-235.

68. Gajewicz, A.; Puzyn, T.; Rasulev, B.; Leszczynska, D.; Leszczynski, J. Metal oxide nanoparticles: Size-dependence of quantum-mechanical properties. Nanosci. Nanotechnol. Asia 2011, 1, 53-58.

69. Katritzky, A.R.; Kulshyn, O.V.; Stoyanova-Slavova, I.; Dobchev, D.A.; Kuanar, M.; Fara, D.C.; Karelson, M. Antimalarial activity: A QSAR modeling using CODESSA PRO software. Bioorg. Med. Chem. 2006, 14, 2333-2357.

70. Tetko, I.V. Computing chemistry on the web. Drug Discov. Today 2005, 10, 1497-1500. 
71. Puzyn, T.; Rostkowski, P.; Swieczkowski, A.; Jedrusiak, A.; Falandysz, J. Prediction of environmental partition coecients and the henrys law constants for 135 congeners of chlorodibenzothiophene. Chemosphere 2006, 62, 1817-1828.

72. Homepage of Molecular Operating Environment (MOE): Chemical Computing Group. Available online: http://www.chemcomp.com (accessed on 1 October 2010).

73. Liu, R.; Zhang, H.Y.; Ji, Z.X.; Rallo, R.; Xia, T.; Chang, C.H.; Nel, A.; Cohen, Y. Development of structure-activity relationship for metal oxide nanoparticles. Nanoscale 2013, 5, 5644-5653.

74. Toropov, A.A.; Toropova, A.P. Quasi-QSAR for mutagenic potential of multi-walled carbon-nanotubes. Chemosphere 2015, 124, 40-46.

75. Wang, S.S.; Cheng, Y.B.; Lin, Y.C.; Liaw, C.C.; Chang, J.Y.; Kuo, Y.H.; Shen, Y.C. Nitrogen-containing diterpenoids, sesquiterpenoids, and nor-diterpenoids from Cespitularia taeniata. Mar. Drugs 2015, 13, 5796-5814.

76. Toropova, A.P.; Toropov, A.A.; Benfenati, E.; Puzyn, T.; Leszczynska, D.; Leszczynski, J. Optimal descriptor as a translator of eclectic information into the prediction of membrane damage: The case of a group of $\mathrm{ZnO}$ and $\mathrm{TiO}_{2}$ nanoparticles. Ecotoxicol. Environ. Saf. 2014, 108, 203-209.

77. Toropova, A.P.; Toropov, A.A.; Rallo, R.; Leszczynska, D.; Leszczynski, J. Optimal descriptor as a translator of eclectic data into prediction of cytotoxicity for metal oxide nanoparticles under different conditions. Ecotoxicol. Environ. Saf. 2015, 112, 39-45.

78. Hamilton, R.F.; Wu, N.; Porter, D.; Buford, M.; Wolfarth, M.; Holian, A. Particle length-dependent titanium dioxide nanomaterials toxicity and bioactivity. Part. Fibre Toxicol. 2009, 6, 1-11.

79. Frohlich, E. The role of surface charge in cellular uptake and cytotoxicity of medical nanoparticles. Int. J. Nanomed. 2012, 7, 5577-5591.

80. Rogers, S.N. Toxicological Effects of Cerium Oxide Nanoparticle Aggregates on Caenorhabditis elegans. Master Thesis, Marshall University, Huntington, WV, USA, 2013.

81. Arvizo, R.R.; Miranda, O.R.; Thompson, M.A.; Pabelick, C.M.; Bhattacharya, R.; Robertson, J.D.; Rotello, V.M.; Prakash, Y.S.; Mukherjee, P. Effect of nanoparticle surface charge at the plasma membrane and beyond. Nano Lett. 2010, 10, 2543-2548.

82. Walkey, C.D.; Chan, W.C. Understanding and controlling the interaction of nanomaterials with proteins in a physiological environment. Chem. Soc. Rev. 2012, 41, 2780-2799.

83. Raemy, D.O.; Grass, R.N.; Stark, W.J.; Schumacher, C.M.; Clift, M.J.D.; Gehr, P.; Rothen-Rutishauser, B. Effects of flame made zinc oxide particles in human lung cells-A comparison of aerosol and suspension exposures. Part. Fibre Toxicol. 2012, 9, 1-18.

84. Calderon-Aparicio A.; Strasberg-Rieber, M.; Rieber M. Disulfiram anti-cancer efficacy without copper overload is enhanced by extracellular $\mathrm{H}_{2} \mathrm{O}_{2}$ generation: Antagonism by tetrathiomolybdate. Oncotarget 2015, 6, 29771-29781.

85. Ge, Y.; Zhang, Y.; Xia, J.; Ma, M.; He, S.; Nie, F.; Gu, N. Effect of surface charge and agglomerate degree of magnetic iron oxide nanoparticles on $\mathrm{KB}$ cellular uptake in vitro. Colloids Surf. B 2009, 73, 294-301.

86. Osaka, T.; Nakanishi, T.; Shanmugam, S.; Takahama, S.; Zhang, H. Effect of surface charge of magnetite nanoparticles on their internalization into breast cancer and umbilical vein endothelial cells. Colloids Surf. B 2009, 71, 325-330. 
87. Bellanger, X.; Billard, P.; Schneider, R.; Balan, L.; Merlin, C. Stability and toxicity of ZnO quantum dots: Interplay between nanoparticles and bacteria. J. Hazard. Mater. 2015, 283, 110-116.

88. Kumar, V.; Kumari, A.; Guleria, P.; Yadav, S.K. Evaluating the toxicity of selected types of nanochemicals. Rev. Environ. Contam. Toxicol. 2012, 215, 39-121.

89. Linkov, I.; Steevens, J.; Adlakha-Hutcheon, G.; Bennett, E.; Chappell, M.; Colvin, V.; Davis, J.M.; Davis, T.; Elder, A.; Foss Hansen, S.; et al. Emerging methods and tools for environmental risk assessment, decision-making, and policy for nanomaterials: Summary of NATO advanced research workshop. J. Nanopart. Res. 2009, 11, 513-527.

(C) 2015 by the authors; licensee MDPI, Basel, Switzerland. This article is an open access article distributed under the terms and conditions of the Creative Commons Attribution license (http://creativecommons.org/licenses/by/4.0/). 